\title{
Atomic Scale Dynamic Process of Cu Oxidation Revealed By Correlated in situ Environmental TEM and DFT Simulations
}

\author{
Meng Li ${ }^{1}$, Matthew T. Curnan ${ }^{1}$, Michael A. Cresh-Sill ${ }^{1}$, Stephen D. House ${ }^{1,2}$, Wissam A. Saidi ${ }^{1,3}$, Judith \\ C. Yang ${ }^{1,2,4^{*}}$ \\ ${ }^{1}$ Department of Chemical and Petroleum Engineering, University of Pittsburgh, Pittsburgh, PA (USA) \\ ${ }^{2}$ Environmental TEM Catalysis Consortium (ECC), University of Pittsburgh, Pittsburgh, PA (USA). \\ ${ }^{3}$ Department of Mechanical Engineering \& Materials Science, University of Pittsburgh, Pittsburgh, PA \\ (USA) \\ ${ }^{4}$ Department of Physics and Astronomy, University of Pittsburgh, Pittsburgh, PA (USA) \\ * Corresponding author: judyyang@ pitt.edu
}

Surface oxidation of metals and alloys is omnipresent in oxidizing environments, such as air or humid conditions. The oxidation of metals and alloys not only causes problems like corrosion and degradation, but could also be used in applications such as catalysts and transistors. With the increase of application demands toward the nanoscale, a fundamental understanding of the atomic mechanisms responsible for surface oxidation is essential for the prevention, prediction, and controlled growth of metal oxide. Ample studies on the bulk oxidation of metals and alloys using bulk corrosion experimental approaches, such as Thermogravimetric Analysis (TGA), and on the initial interaction and reconstruction of metal surfaces with oxygen using surface science approaches, including Scanning Tunneling Microscopy (STM), have been completed. However, the nucleation and growth of metal oxides is much less investigated, especially at the atomic scale, since neither of these approaches can evaluate these dynamic processes. The flourishing of the in situ TEM technique, especially the development of Environmental TEM (ETEM), is very suitable and promising for narrowing this knowledge gap.

In this work, using correlated in situ Environmental TEM (ETEM) (Hitachi H-9500 operating at 300 $\mathrm{keV}$ with home-made gas delivery system) and atomistic simulations, [1] the atomic-scale dynamic processes of $\mathrm{Cu}_{2} \mathrm{O}$ island growth on $\mathrm{Cu}$ substrates under $\mathrm{O}_{2}$ oxidation were investigated. Singlecrystalline $\mathrm{Cu}(100)$ thin films with a thickness of $60 \mathrm{~nm}$ were first reduced inside the ETEM at $600{ }^{\circ} \mathrm{C}$ under $\mathrm{H}_{2}$, in order to reduce native oxides and create holes with (100) and (110) facets. In situ oxidation experiments were then carried out on these facets at $300{ }^{\circ} \mathrm{C}$ under $\mathrm{O}_{2}$. [2] Epitaxial $\mathrm{Cu}_{2} \mathrm{O}(100)$ nanoislands were observed to grow on $\mathrm{Cu}$ facets via monolayer-by-monolayer growth along $\mathrm{Cu}_{2} \mathrm{O}(110)$ planes, instead of along $\mathrm{Cu}_{2} \mathrm{O}(100)$ planes, regardless of $\mathrm{Cu}$ substrate orientation (Figure 1). This result contradicts previous theoretical predictions stating that epitaxial metal oxides grow along metal surfaces, namely via inward diffusion of oxygen or outward diffusion of metal through the oxide. Correlated Density Functional Theory (DFT) simulations on the surface and diffusion energies during $\mathrm{Cu}_{2} \mathrm{O}$ growth on various $\mathrm{Cu}_{2} \mathrm{O}$ surface orientations and terminations were carried out. Our DFT results show that monolayer formation of $\mathrm{Cu}_{2} \mathrm{O}$ along $\mathrm{Cu}_{2} \mathrm{O}(110)$ was both thermodynamically and kinetically preferred over that of $\mathrm{Cu}_{2} \mathrm{O}(100)$ during $\mathrm{Cu}_{2} \mathrm{O}$ growth, which explains the observed phenomenon. To better extract the atomic scale dynamic process of $\mathrm{Cu}_{2} \mathrm{O}$ growth, an automated data analysis approach was developed using in-house codes made with ImageJ and MATLAB. The atomic scale growth trajectories of the $\mathrm{Cu}_{2} \mathrm{O}(110)$ layers were then extracted and analyzed, as is shown in Figure 2. Further analysis and theoretical explanations of the observed growth trajectories are underway. Our results will enhance the understanding of surface oxidation and will also provide insights into understanding initial oxide growth mechanisms of other metal/alloy systems. [3] 


\section{References:}

[1] Curnan, M. T. et al. J. Phys. Chem. C 123, 1, 452-463 (2018). DOI: 10.1021/acs.jpcc.8b08944

[2] Li, M. et al. Microsc. Microanal. 24, 262-263 (2018). DOI: 10.1017/S1431927618001800

[3] The authors acknowledge funding from the National Science Foundation (NSF) grants DMR-

1410055, DMR-1508417, DMR-1410335, and DMREF CHE-1534630, as well as support from Hitachi High Technologies and technical assistance from the Nanoscale Fabrication and Characterization Facility (NFCF) in the Petersen Institute of Nano Science and Engineering (PINSE) at the University of Pittsburgh. The ECC is funded through the University of Pittsburgh and Hitachi High Technologies.
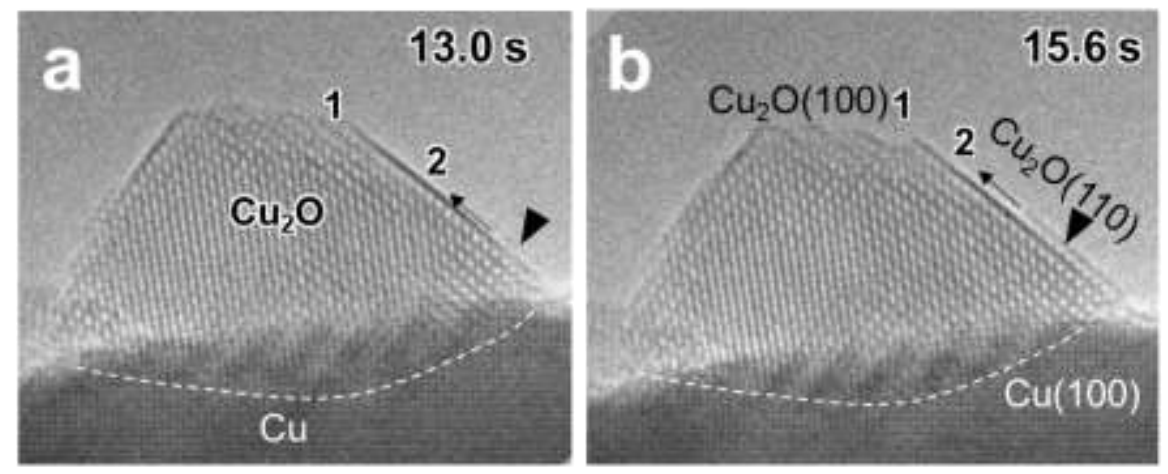

Figure 1. In situ observation of layer-by-layer $\mathrm{Cu}_{2} \mathrm{O}$ growth along $\mathrm{Cu}_{2} \mathrm{O}(110)$ under $0.3 \mathrm{~Pa} \mathrm{O}_{2}$ at $300^{\circ} \mathrm{C}$.
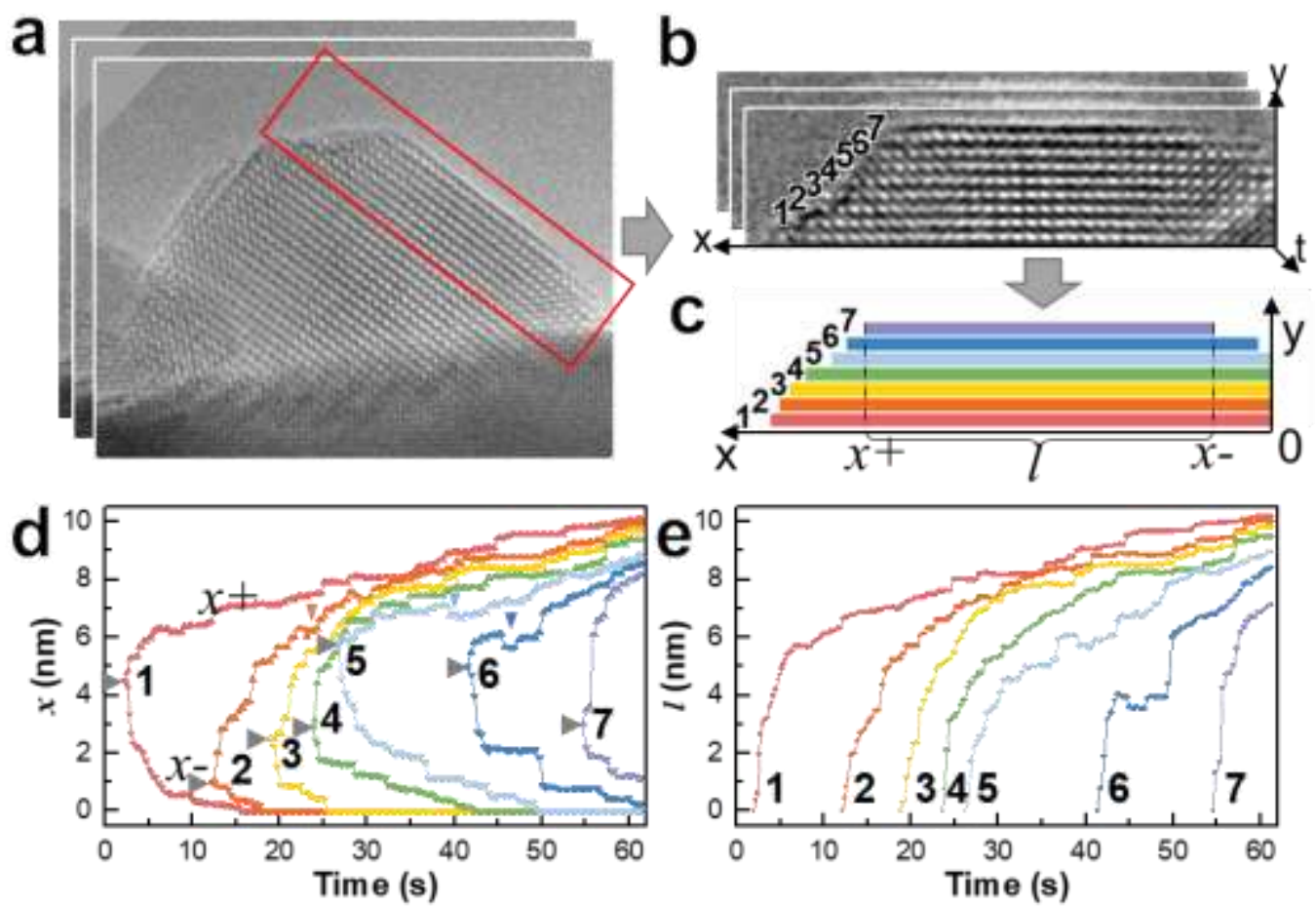

Figure 2. Processing of in situ HRTEM movie to extract atomic scale growth trajectories. a) Image stacks of the HRTEM movie after drift-correction. b) Enhanced and cropped image stacks for growth trajectory extraction. c) Schematic of the one frame of the extracted layers and the corresponding growth trajectories of each layer, namely in terms of (d) edge positions and (e) total length. 